\title{
TRATAMIENTO DE LA LITIASIS RENAL CON BIFOSFONATOS.
}

\author{
Miguel Arrabal Martín, Francisco Valle Díaz de la Guardia, Antonio Jiménez Pacheco, Víctor \\ López León, Miguel Ángel Arrabal Polo y Armando Zuluaga Gómez.
}

Servicio de Urología. Hospital Universitario San Cecilio. Granada. España.

\begin{abstract}
Resumen.- OBJETIVO: El tratamiento actual de la hipercalciuria aún es controvertido, no recomendándose la restricción de calcio ya que puede producir un balance negativo con importantes consecuencias en el metabolismo óseo. En adultos, el uso de bifosfonatos (alendronato sódico) ha mostrado buena respuesta. Los bifosfonatos son análogos sintéticos del pirofosfato endógeno, el pirofosfato es la forma más simple del fosfato, Fleisch en 1968 demostró que el pirofosfato inorgánico inhibe la precipitación del fosfato cálcico. La diferencia entre los diferentes bifosfonatos está en el margen de seguridad entre su efecto inhibidor de la resorción ósea y el efecto inhibidor de la mineralización, su aplicación más difundida ha sido como inhibidores de la resorción ósea. El objetivo de este trabajo, es analizar el efecto clínico y bioquímico de los bifosfonatos en enfermos con hipercalciuria, osteopenia y litiasis renal.
\end{abstract}

MÉTODOS: En el periodo de 1996-2006 hemos tratado 25 casos de litiasis renal recidivante asociada a hipercalciuria y perdida de masa ósea primaria o secundaria. En todos los casos se ha realizado tratamiento con alendronato sódico y aporte oral de calcio equivalente a 1000-1200 mg/día. Analizamos, tolerancia y adherencia al tratamiento, efectos secundarios, efectos bioquímicos en sangre y orina, efectos sobre mineralización ósea y evolución de la actividad litiásica antes y después del tratamiento.

RESULTADOS: Todos los pacientes han seguido las recomendaciones de administración del fármaco lalendronato sódico $10 \mathrm{md} /$ día o $70 \mathrm{mg} / \mathrm{semana}$ ), han manifestado buena tolerancia y no se han producido efectos secundarios relevantes ni abandonos del tratamiento. En el $76 \%$ de los casos se ha producido remisión de la actividad litógena y en el $24 \%$ se ha reducido la actividad litiásica, en todos los casos ha aumentado la mineralización ósea.

CONCLUSIONES: En este grupo seleccionado de pacientes con litiasis recidivante y osteopenia, el tratamiento con bifosfonatos solos o asociados a tiacidas ha obtenido buenos resultados en el control de la litiasis renal y desmineralización ósea. La ampliación de sus indicaciones debe analizarse en un estudio randomizado multicéntrico.

Palabras clave: Litiasis renal. Hipercalciuria. Bifosfonatos.

Summary.- OBJECTIVES: Current treatment of hypercalciuria is still controversial, not being recommended calcium restriction since it may cause a negative balance with important consequences to bone metabolism. In adults, the use of biphosphonates (sodium alendronate) has shown a good response. Biphosphonates are syn- 
thetic analogs of the endogenous pirophosphate. Pirophosphate is the simplest form of phosphate. In 1968, Fleisch demonstrated that inorganic pirophosphate inhibits the precipitation of calcium phosphate. The differences between various biphosphonates are in the safety margin between their inhibitor effect for bone resorption and the inhibitor effect for mineralization; bone resorption inhibition has been their most widely spread application. The objective of this work is to analyze the clinical and biochemical effect of biphosphonates in patients with hypercalciuria, osteopenia and renal lithiasis.

METHODS: From 1996 to 2006 we treated 25 cases of recurrent renal lithiasis associated with hypercalciuria and primary or secondary bone mass loss. All cases were treated with sodium alendronate and oral calcium (1000-1200 mg/day). We analyze tolerance and treatment compliance, side effects, biochemical effects on blood and urine, effect on bone mineralization, and the outcome of lithiasic disease before and after treatment.

RESULTS: All patients have followed the recommendations for the administration of the drug (sodium alendronate $10 \mathrm{mg} /$ day or $70 \mathrm{mg} /$ week), have had good tolerance without relevant side effects, and no one quit treatment.

$76 \%$ of the cases have had remission of the lithogenesis activity and 24\% reduction, and all cases have had an increase of bone mineralization.

CONCLUSIONS: In this group of selected patients with recurrent lithiasis and osteopenia the treatment with biphosphonates alone or associated with thiazide diuretics has given good results in renal lithiasis control and bone demineralization. The extension of indications should be analyzed in a multicentric randomized study.

Keywords: Renal lithiasis. Hypercalciuria.

Biphosphonates.

\section{INTRODUCCIÓN}

El pirofosfato es la forma más simple del fosfato, Fleisch en 1968 demostró que el pirofosfato inorgánico inhibe la precipitación del fosfato cálcico y por lo tanto, impide la formación de cristales de fosfato cálcico in vitro, también se demostró in vivo que los pirofosfatos tenían capacidad de inhibir la calcificación (1).

Tras la introducción de los primeros pirofosfatos se observó que estos compuestos eran hidrolizados por las fosfatasas a nivel intestinal y por tanto inactivos cuando se administraban por vía oral y obli- gaban a su administración por vía intravenosa. Esto llevó a estudiar análogos, que tuvieron características fisicoquímicas similares pero que fueran resistentes a la hidrólisis y no fueran degradados metabólicamente. Así, se llegó a los bifosfonatos, análogos sintéticos del pirofosfato endógeno del que se diferencian por presentar un enlace P-C-P en lugar de P-O-P, que los hace resistentes a las fosfatasas y por tanto, más estables y con gran afinidad por los cristales de hidroxiapatita óseos, y se caracterizan por una baja absorción intestinal (1-5\% de la dosis), pero con una elevada captación y retención a nivel óseo. Se estima que aproximadamente entre el $20 \%$ y el $80 \%$ es captado por el hueso y el resto eliminado por el riñón. Su vida media plasmática es de una hora, pero su permanencia una vez captado por el hueso es de años, ya que no son susceptibles de degradación enzimática por las pirofosfatasas óseas.

Hasta la fecha, han sido sintetizados numerosos bifosfonatos, mediante variaciones de los sustituyentes en los carbonos, de primera, segunda y tercera generación. Según su mecanismo de acción se clasifican en dos grandes grupos: A) No nitrogenados (clodronato, etidronato), estructuralmente semejantes al pirofosfato, para su acción deben ingresar en la célula, donde al acumularse inhiben ciertas enzimas intracelulares, con efectos deletéreos en la función y sobrevida del osteoclasto. B) Nitrogenados (pamidronato, alendronato, ibandronato, zolendronato, etc.), ejercen su acción inhibiendo enzimas de la vía del mevalonato, impidiendo de esta manera la biosíntesis de compuestos isoprenoides, esenciales en la regulación de procesos celulares influyentes en la función y sobrevida del osteoclasto. Ambos grupos conllevan a la muerte celular programada del osteoclasto (apoptosis). Los bifosfonatos también pueden actuar indirectamente estimulando la formación de precursores de los osteoblastos, incrementando su número y diferenciación y permitiendo que estas células liberen sustancias que inhiban a los osteoclastos (2). Otros inhiben la producción de interleukina 6.

La diferencia entre los diferentes bifosfonatos está en el margen de seguridad entre su efecto inhibidor de la resorción ósea y el efecto inhibidor de la mineralización, que es de 1_1 para el etidronato y puede llegar a 1000_1 para el alendronato (Tabla I).

Su aplicación más difundida ha sido como inhibidores de la resorción ósea, en enfermedades donde la actividad osteoclástica está exacerbada como: Paget óseo, metástasis de cáncer de mama, mieloma múltiple e hipercalcemia maligna. Actualmente, diversos bifosfonatos han quedado establecidos y aprobados como tratamiento efectivo para la osteoporosis post-menopáusica y secundaria a otras 
causas. También se ha preconizado su empleo en casos de calcificación distrófica, miositis osificante y en la osificación heterotópica, dados sus efectos en ratas sobre la prevención de calcificaciones aórticas, renales y dérmicas inducidas por altas dosis de vitamina $D$ o de las calcificaciónes periarticulares observadas en la artritis experimental. Igualmente se ha utilizado en odontología, tanto para la prevención de los cálculos dentales, como en la periodontitis y en la pérdida del hueso alveolar (3).

En otros campos podrían tener efecto sobre el síndrome de la distrofia simpática refleja, en la osteoartritis, así como en enfermedades del metabolismo óseo hereditario que incluyen la osteogénesis imperfecta, la displasia fibrosa, la mastocitosis sistémica, la displasia fibrosa, la enfermedad de Gaucher, la hiperostosis esternocostoclavicular y la osteopatía hipertrófica (4-6).

\section{Tratamiento de la hipercalcemia con bifosfonatos}

Los bifosfonatos son los agentes de elección para el tratamiento inicial y a largo plazo de la hipercalcemia (7). Entre las ventajas de su uso en relación con otros fármacos antirreabsortivos figura su acción más prolongada y pronunciada con mejor tolerancia y margen de seguridad, deben administrarse por vía intravenosa. (Clodronato, $4-6 \mathrm{mg} / \mathrm{kg}$ de peso en 2 5 horas durante 3-5 días o Ácido Zoledrónico, 4-8 $\mathrm{mg}$.

\section{Tratamiento médico del hiperparatiroidismo primario con bifosfonatos}

Los bifosfonatos en el HPT disminuyen la calcemia y mejoran los parámetros de resorción ósea, elevación transitoria de la PTH al iniciar el tratamiento (8).Se han utilizado los bifosfonatos tanto para el tratamiento de la hipercalcemia aguda en el seno de un HPT como para los casos de carcinoma paratiroideo inoperable. En estos casos de HPT, el efecto sobre la hipercalcemia desaparece rápidamente tras la supresión del tratamiento.

\section{Tratamiento de la osteoporosis con bifosfonatos}

Los bifosfonatos tienen un papel relevante en el tratamiento de la osteoporosis, no sólo disminuyendo los marcadores del remodelado óseo a través de su acción antireabsortiva, sino que también aumentan la densidad mineral ósea y sobre todo reducen la incidencia de fracturas osteoporóticas. Los bifosfonatos utilizados y aprobados en España para el

TABLA I . POTENCIA RELATIVA DE LOS Bifosfonatos (P.R.B.) .

\begin{tabular}{|c|l|l|}
\hline \multicolumn{1}{|c|}{ Modificación química } & \multicolumn{1}{|c|}{ Bifosfonato } & P.R.B \\
\hline Primera generación & & 1 \\
\hline & Etidronato & 10 \\
\hline Segunda generación & Clodronato & \\
\hline Grupo amino terminal & & 10 \\
\hline & Tiludronato & 100 \\
\hline Tercera generación & Pamidronato & $100-1000$ \\
\hline Lado de cadena cíclica & Alendronato & $1000-10.000$ \\
\hline & Risedronato & $1000-10.000$ \\
\hline & Ibandronato & $1000-10.000$ \\
\hline & Zolendronato & \\
\hline
\end{tabular}


tratamiento de la osteoporosis son: etidronato, alendronato, risedronato y vandronato (9).

a) El etidronato fue el primer bisfosfonato que se utilizó en clínica, en su indicación para osteoporosis se utilizó de forma cíclica para evitar defectos en la mineralización de hueso.

b) El alendronato preserva la masa ósea en todo el esqueleto y principalmente en aquellas regiones que tienen mayor riesgo de fracturas, no condiciona alteraciones en la mineralización del hueso, es eficaz a dosis de $10 \mathrm{mg} /$ día, se toma diariamente y es bien tolerado (10). La eficacia demostrada de tratamientos con dosis oral de $70 \mathrm{mg}$ de alendronato una vez por semana (11) puede facilitar el cumplimiento del tratamiento. En varones con osteoporosis el alendronato aumenta significativamente la densidad mineral ósea de cadera, y columna, disminuye los marcadores de recambio óseo y la tasa de fracturas.

c) El risedronato preserva la masa ósea en todo el esqueleto y principalmente en aquellas regiones que tienen mayor riesgo de fracturas, no condiciona alteraciones en la mineralización del hueso, se toma diariamente y es bien tolerado, a dosis de $5 \mathrm{mg} /$ día demostró reducción de nuevas fracturas vertebrales en un $41-49 \%$, la reducción de fracturas no vertebrales fue del $33-39 \%$ (muñeca, cadera, húmero, clavícula, pelvis y pierna) (12).

\section{Tratamiento de la hipercalciuria con bifosfonatos}

El tratamiento actual de la hipercalciuria aún es controvertido, basado en una dieta baja en sal y

TABLA II. DISTRIBUCIÓN CASOS DE LITIASIS RENAL.

\begin{tabular}{|l|c|}
\hline \multicolumn{1}{|c|}{ DIAGNÓSTICO } & № de Casos \\
\hline Acidosis tubular renal & 5 \\
\hline Osteopenia & 7 \\
\hline Enfermedad de Paget ósea & 1 \\
\hline Hipercalciuria renal & 6 \\
\hline Hiperparatiroidismo primario & 2 \\
\hline Hipofosfatasia & 3 \\
\hline Hipofosfatemia, vitamina D elevada & 1 \\
\hline
\end{tabular}

restricción de proteínas de origen animal, aumento del ejercicio, tiacidas que favorecerían la reabsorción renal tubular distal de calcio e inhibirían la resorción ósea (13-14), no recomendándose la restricción de calcio ya que puede producir un balance negativo con importantes consecuencias en el metabolismo óseo. En adultos, el uso de bifosfonatos (alendronato sódico) ha mostrado buena respuesta (15).

\section{Efectos secundarios de los bifosfonatos}

El efecto colateral descrito con más frecuencia es la intolerancia gastrointestinal para todos los bifosfonatos, incluyendo náuseas, dispepsia, esofagitis e incluso úlcera gastrointestinal, se aconseja su administración con el estómago vacío, 30 minutos antes del desayuno y evitando el decúbito. Los bifosfonatos administrados por vía oral pueden originar hipocalcemias que no son clínicamente significativas excepto en pacientes con hipoparatiroidismo. La administración iv rápida de grandes cantidades de bifosfonatos puede precipitar una insuficiencia renal aguda. A nivel óseo algunos bifosfonatos, especialmente el etidronato, pueden originar inhibición de la mineralización ósea y osteomalacia, por lo que se recomienda su utilización en pautas cíclicas. En estudios experimentales con altas dosis algunos bifosfonatos han mostrado una deficiente reparación de microfracturas y retraso en la consolidación del callo de fractura, aunque no existen datos en humanos (16). Atraviesan la barrera placentaria y afectan al feto, y están contraindicados en embarazo y lactancia.

El objetivo de este trabajo, es analizar el efecto clínico y bioquímico de los bifosfonatos en enfermos con hipercalciuria, osteopenia y litiasis renal.

\section{MATERIAL Y MÉTODOS}

En el periodo de 1996-2006 hemos tratado 25 casos de litiasis renal recidivante, 14 mujeres y 11 hombres con edades entre 25-74 años, periodo de seguimiento medio de 5 años. El diagnóstico etiológico de la litiasis se relaciona con acidosis tubular renal (ATR), osteopenia, enfermedad de Paget ósea, hipercalciuria renal, hiperparatiroidismo, hipofosfatasia, hipofosfatemia (Tabla II).

En todos los casos se ha realizado tratamiento con alendronato sódico $(10 \mathrm{mg} /$ día o $70 \mathrm{mg} / \mathrm{se}$ mana) y aporte oral de calcio equivalente a 1000 $1200 \mathrm{mg} /$ día. En 6 casos de hipercalciuria renal se ha asociado hidroclorotiacida, $50 \mathrm{mg} /$ día y en 5 casos de ATR se ha asociado citrato potásico, 20 $\mathrm{mEq} / 8$ horas. 
Hemos recogido antecedentes clínicos y terapéuticos por litiasis y realizado estudio bioquímico plasmático (creatinina, calcio, fósforo, fosfatasa alcalina, PTH, Vitamina D) y de orina (calciuria postayuno y en 24 horas) antes de iniciar el tratamiento y durante el periodo de seguimiento cada 6 meses. Densitometría ósea al inicio del tratamiento y a los 2 años. Estudio radiológico y ecográfico renal, cuantificando número de cálculos y masa litiasica inicial, evaluación radiológica y/o ecográfica cada 6 meses.

Analizamos, tolerancia y adherencia al tratamiento, efectos secundarios, efectos bioquímicos en sangre y orina, efectos sobre mineralización ósea y evolución de la actividad litiásica antes y después del tratamiento con alendronato sódico.

\section{RESULTADOS}

Todos los pacientes han seguido las recomendaciones de administración del fármaco, han manifestado buena tolerancia y no se han producido efectos secundarios relevantes ni abandonos del tratamiento. La dosis diaria de $10 \mathrm{mg}$ se ha sustituido en todos los casos por la dosis semanal de $70 \mathrm{mg}$.

Los 5 casos de ATR presentaban al inicio del tratamiento litiasis renal múltiple bilateral, pérdida de masa ósea (T score media -3.2 DE), antecedentes de cólicos renales expulsivos y 2 casos habían sido tratados con litotricia extracorpórea, (Figuras 1 y 2). El tratamiento con alendronato y citrato potásico ha controlado la progresión de la enfermedad litiásica, al inicio del tratamiento con alendronato se observó descenso moderado de la calcemia y fosfatemia, descenso de la calciuria de ayuno y de 24 horas, tras 2 años de tratamiento se observó descenso de la fosfatasa alcalina, calcemia y fosfatemia normal, calciuria de ayuno mínima, $6.6 \mathrm{mg} / \mathrm{dl}$, ha aumentado la densidad mineral ósea (T score media -1.5 DE) y ha disminuido al $50 \%$ la masa litiásica inicial.

Los 7 casos de litiasis y osteopenia, presentan antecedentes de litiasis recidivante (2-3 cálculos anuales en los últimos 3 años y nefrocalcinosis en 2 casos) con necesidad de múltiples sesiones de litotricia extracorpórea. El tratamiento con alendronato y aporte oral de calcio ha evitado las recidivas en 5 casos y reduce el número de recidivas en 2 casos (Figuras 3 y 4 ). Al iniciar el tratamiento se observa descenso moderado de calcio y fósforo en sangre con cifras de PTH normales y calciuria de $12 \mathrm{mg} / \mathrm{dl}$, en 2 casos la asociación de tiacidas normaliza las cifras de fósforo y calcio en sangre, la densidad mineral ósea evoluciona de T score media -2.7 a -1.8 DE.

Los 6 casos de hipercalciuria renal, osteopenia y litiasis recidivante corresponden a 4 enfermos con antecedentes de 3-4 episodios de litiasis en dos años tratados con litotricia extracorpórea, tras iniciar tratamiento con tiacidas y alendronato no se han producido recidivas en 3 casos, el $4^{\circ}$ enfermo ha expulsado un cálculo en tres años de evolución. Los otros casos corresponden a 2 mujeres con litiasis papilar y pielocalicial múltiple, tratadas con 10 sesiones de litotricia durante 4 años y paratiroidectomía en un

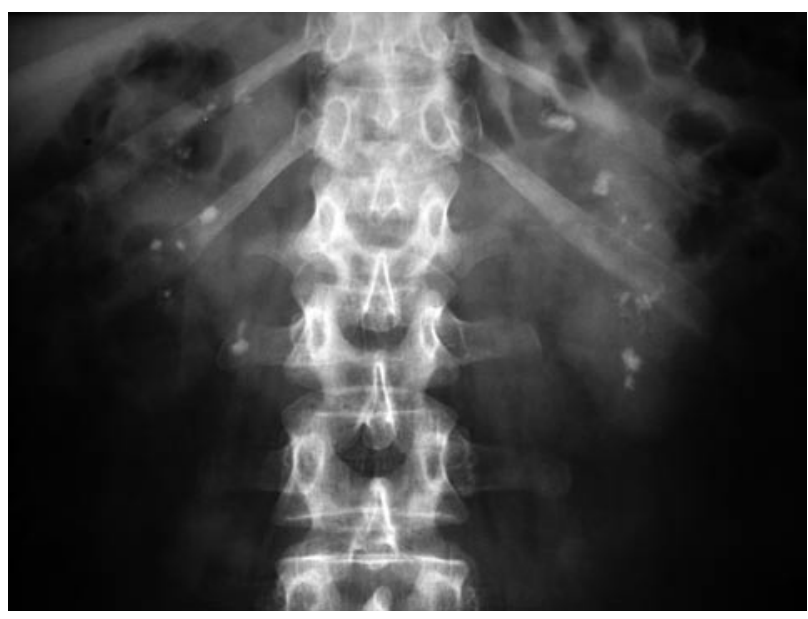

FIGURA 1. Litiasis pielocalicial múltiple bilateral en paciente con ATR.

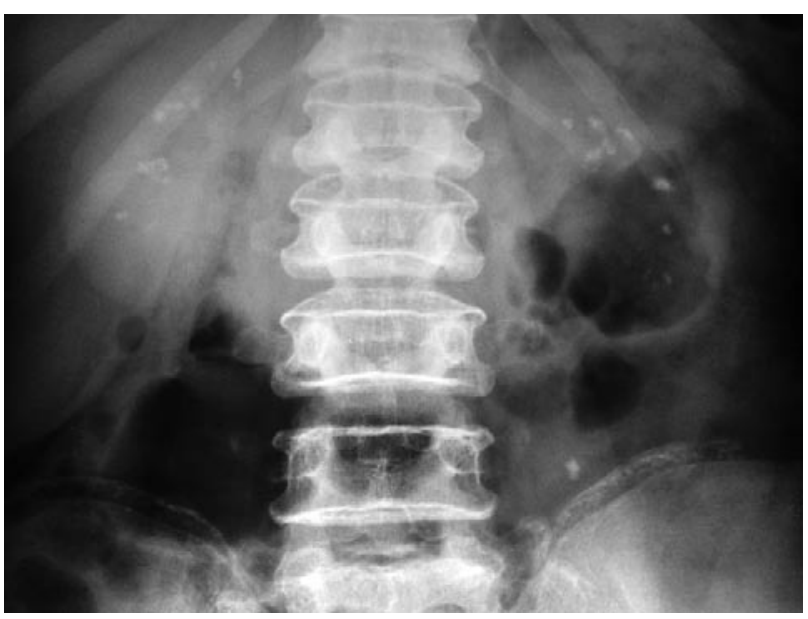

FIGURA 2. Nefrocalcinosis y litiasis, deformidad cuerpos vertebrales en paciente con ATR completa. 
caso por hiperparatiroidismo secundario (Figura 5), tras iniciar tratamiento médico se ha reducido la actividad litógena y la necesidad de nuevas sesiones de litotricia, en 7 años de evolución la masa litiásica se ha reducido al $35 \%$, los parámetros bioquímicos y endocrinos del metabolismo fosfocálcico son normales y ha aumentado la mineralización ósea.

Los 2 casos de HPT, corresponden a una enferma de 74 años que presenta litiasis renal bilateral no obstructiva, se diagnosticó de HPT hipercalcémico (calcemia de $13.5 \mathrm{mg} / \mathrm{dl}$ ), no aceptó tratamiento quirúrgico, el tratamiento con alendronato mantiene las cifras de calcio en rango de $10.5-11.5 \mathrm{mg} / \mathrm{dl}$, con PTH de $155.2 \mathrm{pg} / \mathrm{ml}$ y ha estabilizado la litiasis renal sin aumentar la masa litiásica en tres años. El otro caso corresponde a una mujer de 54 años que presenta litiasis renal izquierda tratada con litotricia y diagnosticada de HPT primario normocalcémico, el tratamiento con bifosfonatos la mantiene asintomática durante 6 años sin cambios bioquímicos ni endocrinos.

Los 3 casos de hipofosfatasia corresponden a tres hombres con historia clínica de litiasis renal bilateral recidivante y osteopenia, que ha requerido tratamiento con litotricia extracorpórea y endoscópi$c a$ en varios episodios. Tras iniciar tratamiento con alendronato se produce remisión completa de la enfermedad litiásica en 2 casos y mejora la densidad mineral ósea.

El caso de enfermedad de Paget ósea, debutó con litiasis renal derecha que fue tratada con una sesión de litotricia persistiendo litiasis residual, el tratamiento

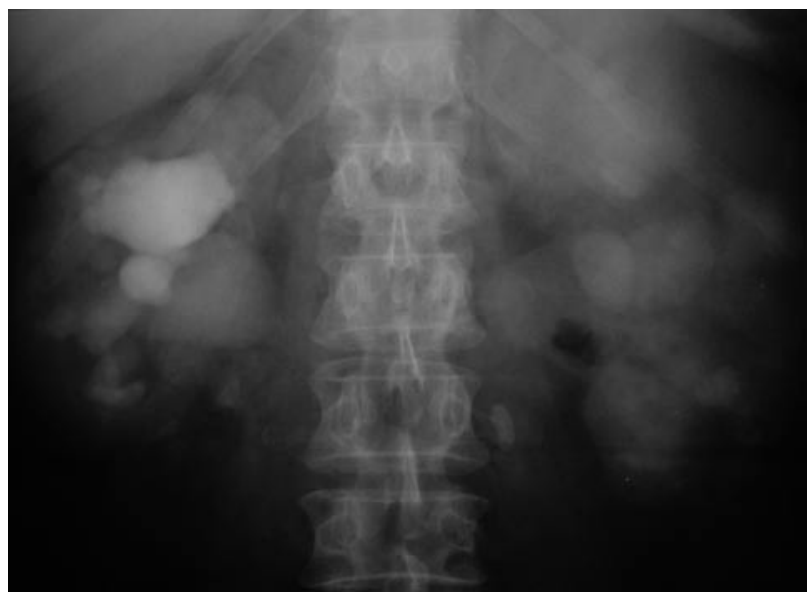

FIGURA 3. Litiasis ureteral bilateral obstructiva. Tratada con litotricia extracorpórea y endoscópica con bifosfonatos ha producido un descenso progresivo de la fosfatasa alcalina, estabilización masa ósea y litiasis residual. El caso de hipofosfatemia y elevación de la vitamina $D$ presenta nefrocalcinosis y antecedentes de cólicos renales expulsivos, la respuesta inicial al tratamiento médico es satisfactoria.

\section{DISCUSIÓN}

Actualmente, a pesar de todos los métodos diagnósticos de que disponemos, el $50 \%$ de los enfermos se diagnostican de hipercalciuria idiopática $(\mathrm{HI})$; este diagnóstico incluye hipercalciurias absortivas, excretoras y resortivas que no se asocian a una patología definida (hiperparatiroidismo primario, sarcoidosis, hipervitaminosis $D$, acidosis tubular renal, etc), no obstante es posible que en muchos casos esté relacionada con aumento del turnover óseo (17). La patogenia de la pérdida de densidad mineral ósea en pacientes con $\mathrm{HI}$ no está del todo aclarada. Se han señalado un gran número de factores causales, entre los que podemos destacar:

a) Hormonas relacionadas con el metabolismo del calcio: el hiperparatiroidismo secundario puede observarse como respuesta a un balance negativo de calcio, bien por defecto en la reabsorción tubular en las formas de predominio renal, o bien por una ingesta reducida de calcio. En ambos casos la pérdida de densidad mineral ósea sería secundaria al aumento de resorción ósea favorecida por la PTH (18) y calcitriol (19).

b) Factores nutricionales: Dietas bajas en calcio pue-

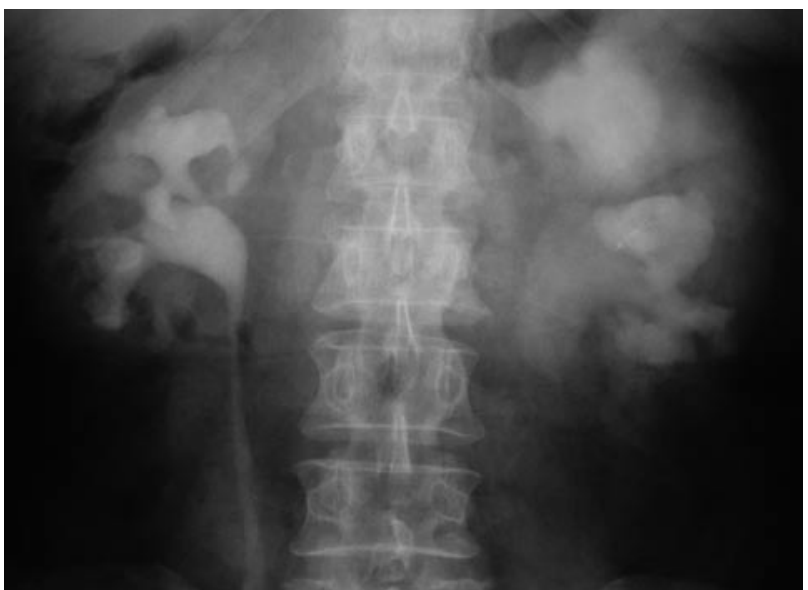

FIGURA 4. Estudio urográfico del paciente figura anterior, 3 años después. 


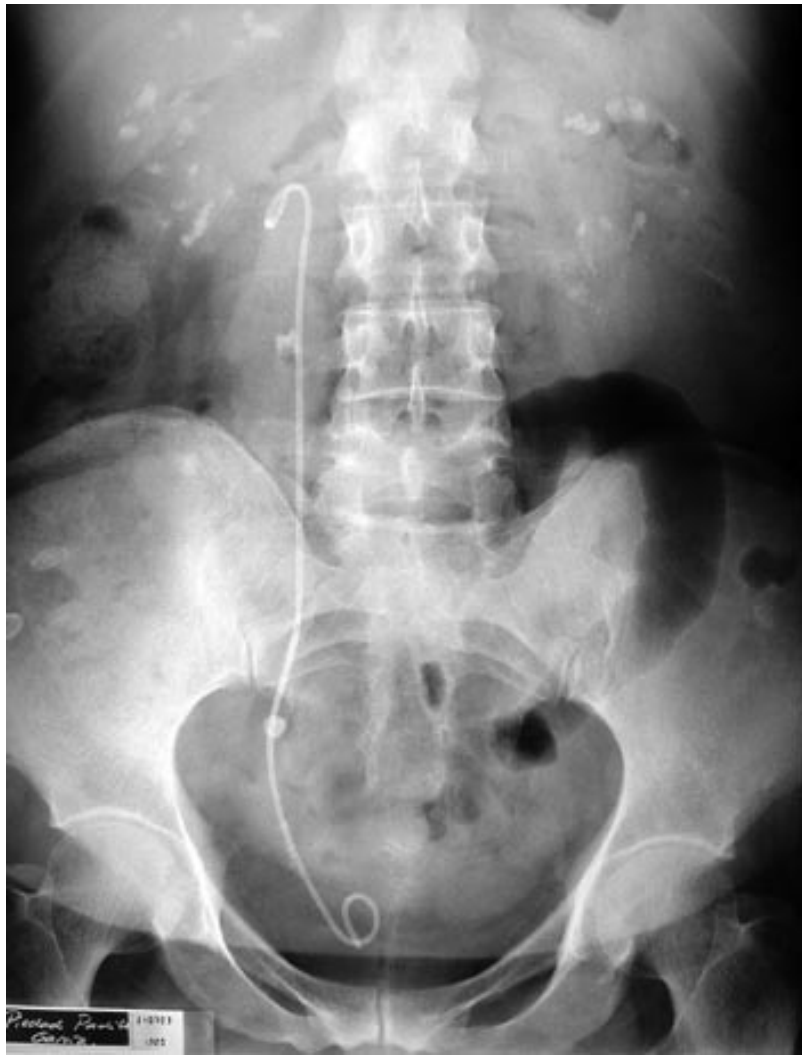

FIGURA 5. Litiasis renoureteral bilateral en paciente con hipercalciuria renal e hiperparatiroidismo secundario, catéter doble jota derecho en la fase de tratamiento con ondas de choque.

den favorecer la osteopenia; además, el exceso en la ingesta de proteínas puede tener un efecto directo sobre la DMO y favorecer la aparición de hipercalciuria, aumentando la acción buffer de hueso debido al intercambio de los iones de calcio por el exceso de iones de hidrógeno que aportaría una dieta rica en proteínas de origen animal (19). Experimentalmente se ha visto que la acidosis metabólica no sólo estimula la actividad osteoclástica sino que también inhibiría la osteoblástica. También se ha visto que la ingesta de sodio aumenta la excreción urinaria de calcio debido a que comparten mecanismos tubulares renales de reabsorción.

c) Citoquinas: En 1990, Pacifici y cols., demostraron la existencia de un aumento de la actividad de la interleuquina-1 B de origen monocitario, que relacionaron, debido a su efecto resortivo, con disminución de $\mathrm{DMO}$ observada en pacientes con $\mathrm{HI}$ que se mantiene en ayunas (20). La síntesis de otras citoquinas como el factor de necrosis tumoral y el factor estimulante de los granulocitos macrófagos también esta aumentada en la $\mathrm{HI}$ (21). d) Otros factores: Algunos autores han encontrado eliminación urinaria de PGE2 aumentada en la HI (22), lo que se trataría de un fenómeno primario de la enfermedad que podría estimular la síntesis de calcitriol y tener un efecto resortivo a nivel óseo. El uso de inhibidores de PG reduce la calciuria en pacientes con HI. También se ha sugerido que el déficit de fosfato puede tener relación con la disminución de la DMO al estimular la síntesis de calcitriol (23), producen hipofosfatemia la alcalosis respiratoria, diarrea crónica, carbohidratos, alcohol, insulina, hipotiroidismo, hipokaliemia, etc, y perdida renal por fuga tubular de fosfatos.

Se ha observado en diferentes estudios una relación significativa entre osteopenia e hipercalciuria, Audran, M. y Zancheta, J.R. detectan osteopenia hasta en el $55 \%$ de los pacientes con hipercalciuria $(24,25)$ con $R R=0.52, p<0.001$, y otros autores observan como enfermos con hipercalciuria y litiasis presentan osteopenia y osteoporosis, $54 \%$ y $14 \%$ respectivamente $(24,26)$. Esta relación no es específica de pacientes diagnosticados de hiperparatiroidismo, si no que también se produce en casos de acidosis metabólica, hipercalciuria renal, hipofosfatemia $(26,27)$. La acidosis metabólica endógena o secundaria a ingesta ácida aumenta la resorción ósea, la pérdida tubular de calcio da lugar a hiperparatiroidismo secundario y la hipercalciuria no dietética en la menopausia se relaciona con litiasis urinaria y osteopenia (28), la hipofosfatemia estimula la producción de calcitriol con lo que aumenta la absorción intestinal de calcio y la resorción ósea. En una serie de 1497 casos, Arrabal, M. y cols. observan que el $38 \%$ presentan hipofosfatemia, asociada a hipercalciuria en el $72 \%$ que corresponde al $24 \%$ de los enfermos con litiasis urinaria (29), Yamakawa y cols. confirman la relación significativa entre alteración del metabolismo del fosfato $(30,31)$, osteopenia y litiasis, $\mathrm{p}<0.001$.

Las tiacidas a nivel renal aumentan la reabsorción tubular distal de calcio, por este efecto hipocalciúrico se utilizan en la profilaxis de la litiasis renal cálcica hipercalciúrica (32). El efecto hipocalciúrico de las tiacidas en tratamientos prolongados (18-24 meses) puede tener una acción limitada con posible retorno de la calciuria en algunos casos a niveles previos al tratamiento, sobre todo en las hipercalciurias absortivas, Fernández y cols. observan este efecto limitante en el $25 \%$ de los pacientes con recuperación posterior de la acción hipocalciúrica (33) a partir de los 24 meses y $\sin$ necesidad de "vacaciones terapéuticas". Hoy se considera a las tiacidas como el tratamiento de elección de la hipercalciuria salvo en la relacionada con hiperparatiroidismo primario $(34,35)$. En un 
estudio sobre "Papel de las tiacidas en la profilaxis de la litiasis cálcica recidivante", se observa como los pacientes que reciben tiacidas presenta menor recurrencia que los del grupo control de forma significativa, con chi-cuadrado experimental de $8.73 \mathrm{lp}$ $=0.003$ ), significación aún mayor en los pacientes con hipercaciuria $(p=0.0008)$. En otro estudio, los autores observan como la eliminación diferida de los fragmentos litiásicos residuales tras la aplicación renal de ondas de choque es mayor en el grupo de pacientes tratados con tiacidas, especialmente si estos presentan hipercalciuria (chi-cuadrado experimental de 20.61, $p<0.0001)(33,36)$.

El efecto hipocalciúrico de las tiacidas da lugar a un balance positivo de calcio que frena la acción de la PTH y la resorción ósea como se ha demostrado en estudios clínicos (37), Legrout-Gerot, I. y cols. demuestran que el efecto de las tiacias a nivel óseo es similar al obtenido con la administración de calcio y vitamina $D(38)$ sin aumentar el riesgo de litiasis, el tratamiento prolongado con vitamina $D$ puede inducir nefrocalcinosis y litiasis (39). Es evidente que en pacientes con osteoporosis y litiasis si sustituimos la vitamina $D$ por tiacidas manteniendo el aporte normal de calcio, realizamos profilaxis de la recidiva litiásica y mantenemos la mineralización del hueso. El efecto del aporte de calcio y vitamina D o tiacidas sobre la mineralización ósea es limitado y en algunos casos insuficiente por lo que debemos recurrir a otros fármacos.

En la osteoporosis, puede estar indicada la asociación de fármacos antiresortivos, en los enfermos con litiasis renal no se debe utilizar calcitonina. Los difosfonatos por vía oral (Alendronato sódico, 70 $\mathrm{mg} / \mathrm{semana}$; Ácido risedrónico, $35 \mathrm{mg} /$ semana) o intravenosa (Ácido zoledrónico) han demostrado su capacidad para prevenir la pérdida de masa ósea, (aumentan un $5 \%$ anual la BMD), disminuyen la incidencia de fracturas, la calcemia y la calciuria, por lo que si es necesario puede asociarse calcio y vitamina D para mejorar la masa ósea. Si se produce hipercalciuria, sustituir el calcio y vitamina $D$ por una tiacida $(17,40,41)$.

Senzaki, H. y cols. han demostrado que el alendronato inhibe la formación de microlitos de fosfato cálcico en cultivos celulares (42), Gupta, M. y cols. en un estudio experimental en ratas observan como los bifosfonatos inhiben la formación de cristales de oxalato cálcico en la vejiga sobre una matriz de zinc (43) por su afinidad por el calcio y/o por su efecto hipocalciúrico, Bushinsky, D.A. y cols. han demostrado que el alendronato desciende la sobresaturación urinaria en ratas hipercalciúricas (44). En un estudio clínico, Weisinger, J.R. y cols. observan como pacientes con marcadores de resorción ósea elevados presentan hipercalciuria, el tratamiento con alendronato normaliza los marcadores de resorción ósea y la calciuria, este efecto hipocalciúrico no se observa en pacientes normocalciúricos (15), Ruml, L.A. y cols. en un ensayo clínico aleatorizado en pacientes con resoción ósea aumentada demuestran que el alendronato desciende la calciuria de forma muy significativa frente a los pacientes no tratados, y el índice de formación del oxalato cálcico y fosfato cálcico (45).

Los bifosfonatos han demostrado su eficacia en la prevención de cálculos dentales, calcificación tisular, arterial, cardiaca, renal $(46,47)$, por lo que se han utilizado en el tratamiento de nefrocalcinosis 48 y en la prevención de cálculos renales. Bone, H.G. y cols. en 1979 observan que, en un grupo de 12 pacientes con litiasis renal cálcica no seleccionado, el tratamiento con bifosfonatos de primera generación durante 30 meses reduce o suprime de forma muy significativa la producción de nuevos cálculos en 7 casos y no hay cambios en 5 casos, 3 pacientes presentan debilidad y dolor osteomuscular (en 2 de ellos se eleva la fosfatasa alcalina y en el otro paciente se produce osteopenia) (49), los autores reconocen la eficacia del bifosfonato en la prevención de la recidiva de la litiasis cálcica pero ante la no efectividad en algunos pacientes y los efectos adversos referidos, consideran su utilidad clínica limitada (50).

Hoy conocemos que los bifosfonatos deben indicarse en pacientes con osteopenia y litiasis renal hipercalciúrica (51) y que los modernos bifosfonatos no producen osteopenia, Watanabe, Y. y cols. en un ensayo clínico aleatorizado en personas sometidas a inmovilización prolongada demuestran que la administración de pamidronato aumenta la formación de hueso y evita la hipercalciuria y formación de cálculos renales de forma muy significativa frente a los casos de dos grupos control (52), no obstante el tratamiento con bifosfonatos no debe sustituir a otros métodos establecidos en la prevención de la litiasis renal cálcica, como regulación de hábitos dietéticos, aumentar el aporte oral de líquidos, ejercicio físico, citrato, alopurinol, tiacidas.

Nosotros asociamos bifosfonatos a las tiacidas en el tratamiento de la litiasis por hipercalciuria de ayuno PTH independiente cuando se eleva la calcemia y/o detectamos osteopenia-osteoporosis. En pacientes con osteopenia-litiasis e hipercalciuria moderada utilizamos sólo bifosfonatos con aporte oral de calcio y asociamos tiacidas si se produce hipocalcemia-hipofosfatemia o para potenciar el efecto óseo de los bifosfonatos. 


\section{BIBLIOGRAFÍA y LECTURAS RECOMENDADAS ( ${ }^{*}$ lectura de interés $y^{* *}$ lectura fundamental)}

1. FLEISCH, H.; RUSSELL, R.G.G.; BISAZ, S. y cols.: "The influence of phyrophosphatase analogues (diphosphonates) on the precipitation and dissolution of calcium phosphate in vitro and in vivo". Calcif. Tissue Res., 10: 15, 1968.

2. WATTS, N.B.: "Pharmacology of agents to treat osteoporosis. Bisphosphonates. Mechanism(s) of action". Favus, M.J. ed. Primer on the metabolic bone diseases ad disorders of mineral metabolism, pag. 278-83. Philadelphia, Lippincott Williams \& Wilkins. 1999.

3. SRIVASTA, T.; ALON, U.S.: "Bisphosphonates: from grandparents to grandchildren". Clin. Pediatr., 38: 687, 1999.

4. ASTROM, E.; SODERHALL, S.: "Beneficial effect of bisphosphonate during five years of treatment of severe osteogenesis imperfecta". Acta Paediatr., 87: 64, 1998.

5. LANE, J.M.; KHAN, S.N.; O'CONNOR, W.J. y cols.: "Bisphosphonate therapy in fibrous dysplasia". Clin. Orthop., 382: 6, 2001.

6. ADAMI, S.; FOSSALUZZA, V.; GATTI, D. y cols.: "Bisphosphonate therapy of reflex sympathetic dystrophy syndrome". Ann. Rheum. Dis., 56: 201, 1997.

7. BODY, J.J.; BARTI, R.; BURCKHARDT, P. y cols.: "Current use of bisphosphonates in Oncology". J. Clin. Oncol., 16: 3890, 1998.

8. PARKER, C.R.; BLACKWELL, P.J.; FAIRBAIRN, K.J. y cols.: "Hosking-D-J. Alendronate in the treatment of primary hyperparathyroidrelated osteoporosis: a 2-year study". J. Clin. Endocrinol. Metab., 87: 4482, 2002.

*9. KARPF, D.B.; SHAPIRO, D.R.; SEEMAN, E. y cols.: "Prevention of nonvertebral fractures by alendronate. A meta-analysis. Alendronate Osteoporosis treatment study groups". JAMA, 277: 1159, 1997.

*10. BLACK, D.M.; CUMMINGS, S.R.; KARPF, D.B. y cols.: "Randomised trial of effect of alendronate on risk of fracture in women with existing vertebral fractures. Fracture intervention trial research group". Lancet, 348: 1535, 1996.

11. RIZZOLI, R.; ROUX, C.; GRESPAN, S. y cols.: "Two year results of once-weekly administration of alendronate $70 \mathrm{mg}$ for the treatment of postmenopausal osteoporosis". Bone, 28: 94, 2001.

12. REGINSTER, J.; MINNE, H.W.; SORENSEN, O.H. y cols.: "Randomized trial of the effects of risedronate on vertebral fractures in women with established postmenopausal osteoporosis. Vertebral Efficacy with Risedronate Therapy (VERT) Study Group”. Osteoporos. Int., 11: 83, 2000.
13. YENDT, E.R.; COHHANIM, M.: "Prevention of calcium stones with thiazides". Kidney Int., 13: 397, 1978.

14. LEMANN, J.R.; GRAY, R.W.; MAIERHOFER, W.J. y cols.: "Hydrochllorothiazide inhibits bone resorption in men depite experimentally elevated serum 1, 25 dihydroxyvitamin D concentrations". Kidney Int., 28: 951, 1985.

**15. WEISINGER, J.R.; ALONZO, E.; MACHADO, C. y cols.: "Role of bones in the physiopathology of idiopathic hypercalciuria: effect of amino-bisphosphonate alendronate". Medicina (B. Aires). 1: 45, 1997.

16. JÓDAR JIMENO, E.; MARTÍNEZ DÍAZ-GUERRA, G.; HAWKINS CARRANZA, F.: "Efectos adversos y contraindicaciones de los bifosfonatos". Rapado Errazti, A. Díaz Curiel, M. editores. Bifosfonatos en las enfermedades del metabolismo óseo y mineral, pag. 113-22. FHOEMO. Madrid, 2002.

**17. ARRABAL MARTÍN, M.; GUTIÉRREZ TEJERO, F.; ZULUAGA GÓMEZ, A.: "Síndrome hipercalcémico y enfermedades óseas. Litiasis urinaria”. Lancina Martín, J.A. Arrabal Martín, M. Enfermedades sistémicas y litiasis urinaria, pag. 15-50. Editorial Visión Net. Madrid, 2004.

18. FUSS, M.; PEPERSACK, T.; VAN GEEL, J.: "Involvement of low-calcium diet in the reduced mineral content in idiopathic renal stone formers". Calcif. Tissue. Int., 46: 9, 1990.

19. HESS, B.; ACKERMAN, D.; ESSING, M. y cols.: "Renal mass and serum calcitriol in male idiopathic calcium renal stone formers: role of protein intake". J. Clin. Endocrinol. Metab., 80: 1916, 1995.

*20. PACIFICI, R.; ROTHSTEIN, M.; RIFAS, L. y cols.: "Increased monocyte interleukin-1 activity and decreased vertebral bone density in patients with fasting idiopathic hypercalciuria". J. Clin. Endocrinol. Metab., 71: 138, 1990.

*21. WEISENGER, J.R.; ALONZO, E.; BELLORIMFONT, E. y cols.: "Possible role of cytokines on the bone mineral loss in idiopathic hypercalciuria”. Kidney Int., 49: 244, 1996.

22. HENRIQUEZ LA ROCHE.; RODRÍGUEZ ITURBE, B.; PARRA, G.: "Increased urinary excretion of PGE2 in patient with idiopathic hypercalciuria: the role of bone”. Kidney Int., 49: 1507, 1996.

23. STEINICHE, T.; MOSEKILDE, L.; CHRISTENSEN, M.S. y cols.: "A histomorphometric determination of iliac bone remodeling in patient with recurrent renal stone formation an idiopathic hypercalciuria”. APMIS, 97: 309, 1989.

24. AUDRAN, M.; BATAILLE, P.; SEBERT, J.L. y cols.: "Bone density in idiopathic hypercalciuria in men. Study by dual photon absorptiometry, X- 
ray computed tomography and histomorphometry". Rev. Rhum. Mal. Osteoartic., 58: 747, 1991.

*25. ZANCHETTA, J.R.; RODRIGUEZ, G.; NEGRI, A.L. y cols.: "Bone mineral density in patients with hypercalciuric nephrolithiasis". Nephron., 73: 557, 1996.

26. CAUDARELLA, R.; VESCINI, F.; BUFFA, A. y cols. "Bone mass loss in calcium stone disease: focus on hypercalciuria and metabolic factors". J. Nephrol., 16: 260, 2003.

27. GIANNINI, S.; NOBILE, M.; SELLA, S. y cols.: "Bone disease in primary hypercalciuria". Crit. Rev. Clin. Lab. Sci., 42: 229, 2005.

**28. TSUJI, H.; UMEKAWA, T.; KURITA, T. y cols.: "Analysis of bone mineral density in urolithiasis patients". Int. J. Urol., 12: 335, 2005.

29. ARRABAL MARTÍN, M.; MIJÁN ORTIZ, J.L.; JIMÉNEZ VERDEJO, A. y cols.: "Hipofosfatemia, hipercalciuria y litiasis”. LXIV Congreso Nacional de Urología. Zaragoza, 26 de mayo de 1999.

30. YAMAKAWA, K.; KAWAMURA, J.: “Analysis of hypophosphatemia in calcium nephrolithiasis". Mol. Urol., 4: 365, 2000.

31. FRIEDLANDER, G.: "Phosphate transport and kidney stones". Bull. Acad. Natl. Med., 189: 309, 2005.

32. CACHAT, F.; BARBEY, F.; DAUDON, M.: "Medical treatment of urinary lithiasis". Rev. Med. Suisse Romande., 124: 461, 2004.

33. FERNÁNDEZ, A.; ARRABAL, M.; GARCÍA, M.J. y cols.: "The role of thiazides in the prophylaxis of recurrent calcium lithiasis". Actas Urol. Esp., 30: 305, 2006.

*34. PEARLE, M.S.; ROEHRBORN, C.G.; PAK, C.Y.: "Meta-analysis of randimized trials for medical prevention of calcium oxalate nephrolithiasis". J. Endourol., 13: 679, 1999.

*35. TISELIUS, H.G.: "Possibilities for preventing recurrent calcium stone formation: principles for the metabolic evaluation of patients with calcium stone disease". BJU Int., 88: 158, 2001.

*36. ARRABAL, M.; FERNÁNDEZ, A.; ARRABALPOLO, M.A. y cols.: "Extracorporeal renal lithotripsy: evolution of residual lithiasis treated with thiazides". Urology, 68: 956, 2006.

37. SUAREZ FERNANDEZ, C.; RUILOPE URIOSTE, L.M.: "Thiazide diuretics and calcium metabolism: role in renal lithiasis and osteoporosis". An. Med. Interna, 13: 401, 1996.

**38. LEGROUX-GEROT, I.; CATANZARITI, L.; MARCHANDISE, X. y cols.: "Bone mineral density changes in hypercalciuretic osteoporotic men treated with thiazide diuretics". Joint Bone Spine, 71: 51, 2004.

39. JACKSON, R.D.; LACROIX, A.Z.: "Calcium plus vitamin D supplementation and the risk of fractures". N. Engl. J. Med., 354: 669, 2006.
**40. FLEISCH, H.: "Diphosphonates: history and mechanisms of action". Metab. Bone Dis. Relat. Res., 3: 279, 1981.

41. MEYTHALER, J.M.; TUEL, S.M.; CROSS, L.L.: "Successful treatment of immobilization hypercalcemia using calcitonin and etidronate". Arch. Phys. Med. Rehabil., 74: 316, 1993.

**42. SENZAKI, H.; YASUI, T.; OKADA, A. y cols.: "Alendronate inhibits urinary calcium microlith formation in a three-dimensional culture model". Urol. Res., 32: 223, 2004.

43. GUPTA, M.; TUNCAY, O.L.; VALDERRAMA, E. y cols.: "Inhibition of calcium oxalate urolithiasis in a rat model of lithogenesis using bisphosphonates". J. Endourol., 11: 1, 1997.

44. BUSHINSKY, D.A.; NEUMANN, K.J.; ASPLIN, J. y cols.: "Alendronate decreases urine calcium and supersaturation in genetic hypercalciuric rats". Kidney Int., 55: 234, 1999.

*45. RUML, L.A.; DUBOIS, S.K.; ROBERTS, M.L. y cols.: "Prevention of hypercalciuria and stoneforming propensity during prolonged bedrest by alendronate". J. Bone Miner. Res., 10: 655, 1995.

46. SKIDER, M.N.; ITOH, M.; IWATSUKI, N. y cols.: "Inhibitory effect of a novel bisphosphonate, TRK-530, on dental calculus formation in rats". J. Periodontol., 75: 537, 2004.

47. VAN DYCK, M.; PROESMANS, W.; VAN HOLLEBEKE, E. y cols.: "Idiopathic infantile arterial calcification with cardiac, renal and central nervous system involvement". Eur. J. Pediatr., 148: 374, 1989.

48. McCREDIE, D.A.; POWELL, H.R.; ROTENBERG, E.: "Diphosphonate therapy in nephrocalcinosis”. Br. J. Urol., 48: 93, 1976.

**49. BONE, H.G.; ZERWEKH, J.E.; BRITTON, F. y cols.: "Treatment of calcium urolithiasis with diphosphonate: efficacy and hazards". J. Urol., 121: 568, 1979.

50. VAIDYANATHAN, S.; WATSON, I.D.; JONSSON, O. y cols.: "Recurrent vesical calculi, hypercalciuria, and biochemical evidence of increased bone resorption in an adult male with paraplegia due to spinal cord injury: is there a role for intermittent oral disodium etidronate therapy for prevention of calcium phosphate bladder stones". Spinal Cord., 43: 269, 2005.

51. HEILBERG, I.P.; MARTINI, L.A.; TEIXEIRA, S.H. y cols.: "Effect of etidronate treatment on bone mass of male nephrolithiasis patients with idiopathic hypercalciuria and osteopenia". Nephron., 79: 430, 1998.

52. WATANABE, Y.; OHSHIMA, H.; MIZUNO, K. y cols.: "Intravenous pamidronate prevents femoral bone loss and renal stone formation during 90-day bed rest." J. Bone Miner. Res., 19: 1771, 2004. 